\title{
Numerical Study on Heat Transfer of Gaseous Nitrogen Thermoregulation System in Thermal Vacuum Chamber
}

\author{
Ying Zhou , Chao He, Bing Bai and Juan Ning \\ Beijing Institute of Spacecraft Environment Engineering, Beijing 100094, China
}

\begin{abstract}
Extensive numerical study on the heat transfer performance of the gaseous nitrogen (GN) thermoregulation shroud surface was conducted in this work. The average heat transfer coefficient was investigated under different shroud length and nitrogen parameters (such as velocity, temperature and mass flow rate). The result shows that the heat transfer performance is affected less by shroud length but largely by mass flow rate. When the mass flow rate is constant, the inlet temperature increases heat transfer coefficient. Finally, dimensionless correlation of the average Nusselt number over shroud surface with Reynolds number and Prandtl number was obtained.
\end{abstract}

\section{Introduction}

The spacecraft on orbit is working in cold and hot alternation and high vacuum situation. In order to keep the spacecraft working reliably, sufficient thermal vacuum tests must be carried out on the ground by simulating the space environment $[1,2]$. The capability of the components, the subsystems and the whole spacecraft to withstand the thermal environment in vacuum chamber is assessed, thereby the design and technics problems are exposed [3, 4].

In a conventional thermal vacuum chamber, the liquid nitrogen in the shroud is usually used as coolant to provide a cold background less than $100 \mathrm{~K}$. And the external heat flow received by spacecraft on orbit is simulated by adding the infrared heaters or electric heating films $[5,6]$. However, most of the heat generated by the heaters is absorbed by liquid nitrogen through the radiation of the device under test (DUT). It increases the consumption of liquid nitrogen and a large amount of electric energy, which is less economical. While the temperature regulating shroud system [7-9] simulating the change of external environment by changing its own temperature is a better alternative. It has the advantages of simple structure, easy operation, good economy and high reliability.

There are many kinds of cooling mediums for thermoregulation shroud, which can be classified into two types: liquid refrigerant and gas refrigerant. The working temperature range of liquid refrigerant is from $193 \mathrm{~K}$ to $423 \mathrm{~K}[10]$. If the temperature is too low, the viscosity of the liquid will increase, leading to the increase of flow resistance, which will reduce the heat transfer efficiency of the refrigerant. While using gaseous nitrogen (GN) as refrigerant can make the shroud temperature continuously adjustable within the range of $93 \mathrm{~K}$ to $423 \mathrm{~K}$ and has a wider application prospect $[11,12]$.

In the present work, an extensive numerical study was conducted focusing on the heat transfer characteristics in gaseous nitrogen thermoregulation shroud. By analysing the heat transfer coefficient on the shroud surface, the effects of the nitrogen flow rate, temperature and the shroud length were investigated. In addition, the average Nusselt number over the shroud surface with consideration of the Reynolds number and Prandtl number of nitrogen were presented.

\section{Methodology}

\subsection{Test facility}

The typical GN thermal regulating system is shown in Fig. 1. A thermo regulated stainless steel cylinder, known as the shroud, is assembled in the thermal vacuum chamber. Two round shrouds close the two ends of the cylinder completely, in order to achieve a uniform temperature field around the device under test (DUT).

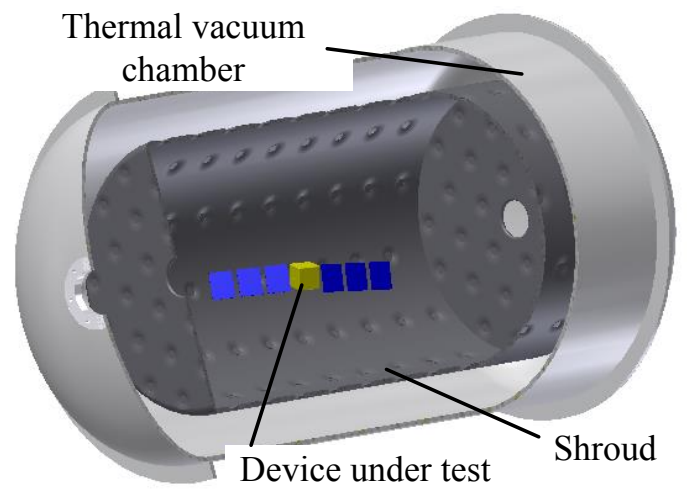

Fig. 1. GN thermoregulation system. 
The shroud consists of two laminated sheets with a space of a few millimetres between them, as shown in Fig. 2. This space is used for the passage of pressurized gaseous nitrogen from the thermal control system. A special black paint is applied on the inner surface of the shroud producing a layer with high emissivity and with low residual mass loss, which maximizes the thermal radiation to DUT in high vacuum condition.

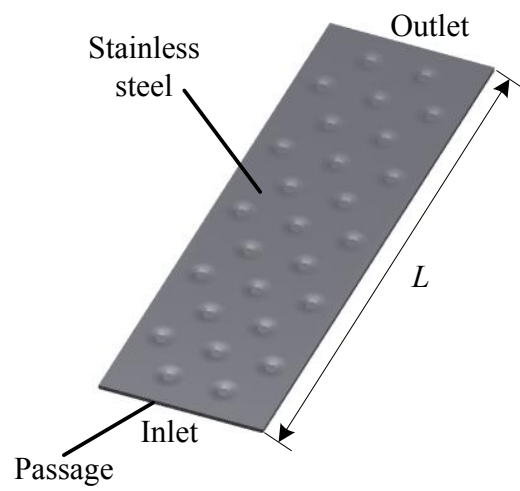

Fig. 2. Shroud details.

\subsection{Thermal analysis}

According to the heat transfer process between shroud surface and pressurized gaseous nitrogen, the total heat $\mathrm{Q}$ transferred from shroud to nitrogen can be calculated by equation(1).

$$
Q=\left(T_{S}-T_{N}\right) h A
$$

where $A$ is the surface area of shroud contacted with nitrogen, $h$ is the average heat transfer coefficient, $T_{S}$ and $T_{N}$ are the average temperature of heat sink and nitrogen respectively, which can be defined as equation(2).

$$
\left\{\begin{array}{l}
T_{S}=\frac{1}{A} \int T d A \\
T_{N}=\frac{T_{N_{-} \text {in }}+T_{N_{-} \text {out }}}{2}
\end{array}\right.
$$

$T$ is the surface temperature of shroud, $T_{N_{-} \text {in }}, T_{N_{-} \text {out }}$ represent the nitrogen temperature into and out of shroud respectively. For nitrogen, the received heat $Q$ from shroud changes the outlet temperature of gas,

$$
Q=\left(T_{N_{-} \text {out }}-T_{N_{-} \text {in }}\right) c_{p} m
$$

where $c_{p}$ is the specific heat of nitrogen, determined by the average temperature $T_{N}$, and $m$ is the mass flow rate of nitrogen.

Therefore, the average temperature of the shroud is dependent not only on the gas conditions such as the temperature, flow rate, and velocity of the high pressure nitrogen but also on the convective heat transfer characteristics of the shroud surface. The former factor can be determined by the shroud design of structure and flow distribution, while the heat transfer coefficient is numerically analyzed using a commercial CFD solver in the present work to obtain general conclusions within a relatively large range of gas conditions.

\section{Numerical solution}

\subsection{Turbulence model}

For the pressurized gaseous nitrogen flowing through the shroud, the control volume of gas element is constrained by the law of mass, momentum and energy conservation. The time-averaged Navier-Stokes equations can be formulated in tensor forms,

$$
\begin{gathered}
\frac{\partial \rho}{\partial t}+\frac{\partial}{\partial x_{i}}\left(\rho u_{i}\right)=0 \\
\frac{\partial}{\partial t}\left(\rho u_{i}\right)+\frac{\partial}{\partial x_{j}}\left(\rho u_{i} u_{j}\right)=\frac{\partial}{\partial x_{j}}\left(-\rho \overline{u_{i}^{\prime} u_{j}^{\prime}}\right)-\frac{\partial p}{\partial x_{i}} \\
+\frac{\partial}{\partial x_{j}}\left[\mu\left(\frac{\partial u_{i}}{\partial x_{j}}+\frac{\partial u_{j}}{\partial x_{i}}-\frac{2}{3} \delta_{i j} \frac{\partial u_{l}}{\partial x_{l}}\right)\right]
\end{gathered}
$$

An additional turbulence stress $\left(-\rho \overline{u_{i}^{\prime} u_{j}^{\prime}}\right)$ is added in equation(5). According to Boussinesq hypothesis [13], this stresses caused by turbulence is related to the mean velocity gradients, which can be written by

$$
-\rho \overline{u_{i}^{\prime} u_{j}^{\prime}}=\mu_{t}\left(\frac{\partial u_{j}}{\partial x_{i}}+\frac{\partial u_{i}}{\partial x_{j}}\right)-\frac{2}{3}\left(\rho k+\mu_{t} \frac{\partial u_{k}}{\partial x_{k}}\right) \delta_{i j}
$$

$k$ is the turbulent kinetic energy of turbulence,

$$
k=\frac{1}{2}\left(\overline{u^{2}}+\overline{v^{2}}+\overline{w^{2}}\right)
$$

$\mu_{t}$ is the viscosity coefficient which is determined by the flow state. In this paper, the standard $k-\varepsilon$ turbulence model [14] is used to calculate the turbulent viscosity coefficient.

$$
\mu_{t}=c_{\mu} \rho k^{2} / \varepsilon
$$

The transport equations of $k$ and epsilon is as follows.

$$
\begin{aligned}
&(\rho k)+\frac{\partial}{\partial x_{i}}\left(\rho k u_{i}\right)=\frac{\partial}{\partial x_{j}} {\left[\left(\mu+\frac{\mu_{t}}{\sigma_{k}}\right) \frac{\partial k}{\partial x_{j}}\right] } \\
&+\mu_{t} S^{2}-\rho \varepsilon-Y_{M} \\
& \frac{\partial}{\partial t}(\rho \varepsilon)+\frac{\partial}{\partial x_{i}}\left(\rho \varepsilon u_{i}\right)=\frac{\partial}{\partial x_{j}} {\left[\left(\mu+\frac{\mu_{t}}{\sigma_{\varepsilon}}\right) \frac{\partial \varepsilon}{\partial x_{j}}\right] } \\
&+C_{1 \varepsilon} \frac{\varepsilon}{k} \mu_{t} S^{2}-C_{2 \varepsilon} \rho \frac{\varepsilon^{2}}{k}
\end{aligned}
$$

\subsection{Mesh}

Structured mesh involving hexahedral elements was generated by ICEM software to simulate the internal flow inside the shroud as shown in Fig. 3. Fine mesh near the shroud surface was presented to get more accurate simulation results. The total mesh quality was above 0.7 .

\subsection{Boundary condition}

Velocity-inlet boundary condition was used for the pressurized nitrogen intake, and pressure-outlet boundary for the exhaust. Since the given computational domain was part of the whole shroud, symmetric boundary condition was used for the two cross sections of the model. A shroud surface was defined as heat-flux 
boundary condition to provide the necessary heat of the heat transfer process. In the calculation, the simple solver was applied. Two order upwind format was used for the discrete format of the control equations to improve the calculation accuracy. The residual and shroud temperature were monitored. The mass and energy conservations of nitrogen flowing in and out of the model were considered as a criteria of calculation convergence.

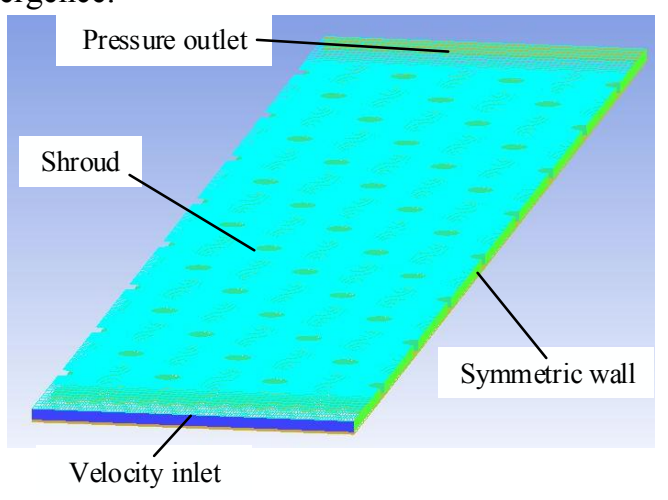

Fig. 3. Mesh of shroud.

\section{Results}
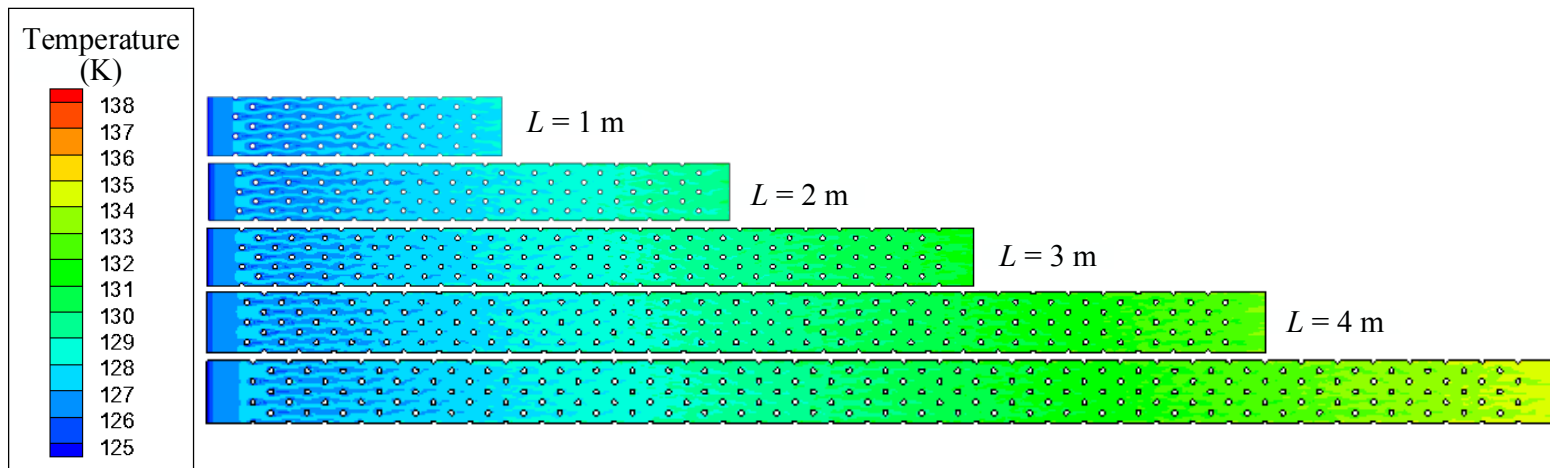

$L=5 \mathrm{~m}$

Fig. 4. Temperature contours of shroud surfaces with different length

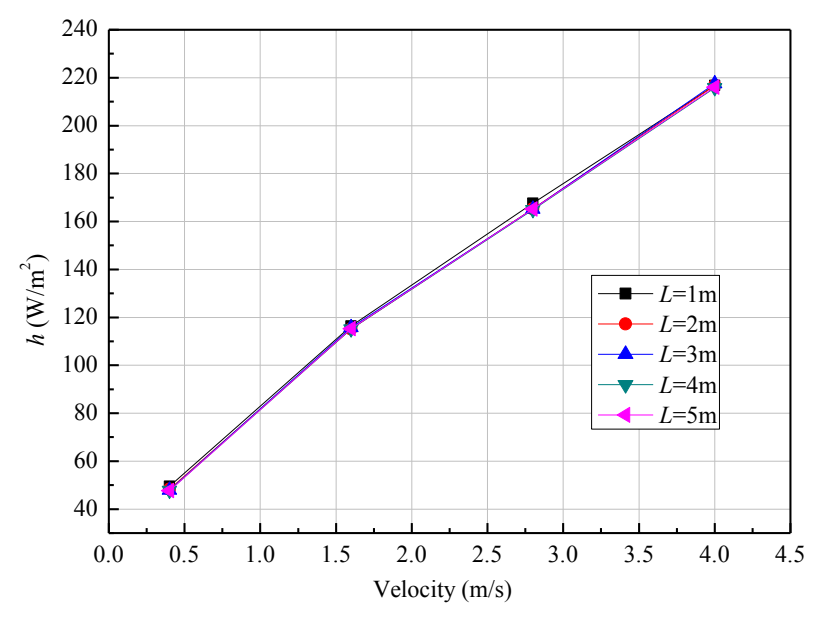

Fig. 5 Average heat transfer coefficient for different shroud

length.

\subsection{Temperature contours}

When the inlet temperature of nitrogen is $T_{N_{-} \text {in }}=123.15$ $\mathrm{K}$ and the inlet velocity is $u=1.6 \mathrm{~m} / \mathrm{s}$, the surface temperature distribution of shroud with different shroud length is shown in Fig. 4.

In order to deeply investigate the heat transfer performance of the shroud, the average heat transfer coefficient $h$ was calculated by equation(1) in section 2.2 .

\subsection{Effect of shroud length}

The average heat transfer coefficient distribution with varying nitrogen velocity under different shroud length $(L=1,2,3,4,5 \mathrm{~m})$ is shown in Fig. 5 to study the effect of the length of different shroud channels. The inlet nitrogen temperature maintained $T_{N_{-} \text {in }}=123.15 \mathrm{~K}$.

The result shows that, the length of shroud flow passage has little effect on the heat transfer performance when the inlet temperature and velocity of nitrogen remain unchanged. It is believed that if the structure of the shroud channel does not change, the heat transfer characteristics will also remain the same due to the constant nitrogen supply condition. This result is quite helpful to the design of thermal vacuum chamber with multiple scales since there is no need to worry about the effect of shroud length on heat transfer.

\subsection{Effect of nitrogen velocity}

As is known from the previous section that the length of the shroud does not affect the heat transfer coefficient, the shroud length of $L=1 \mathrm{~m}$ was applied in the following research to reduce the grid number and expedite the calculation speed.

The distribution of heat transfer coefficient with velocity on shroud surface at different nitrogen temperature is shown in Fig. 6. The results show that the heat transfer coefficient increases with the increase of the velocity. Noting that the higher velocity leads to lager mass flow rate for a constant temperature, the authors believe that the heat transfer performance is mainly affected by mass flow rate. This point can also explain the decrease of heat transfer coefficient with increasing temperature, because the higher the 
temperature is, the lower the gas density is, the smaller the flow rate is when the velocity remains constant.

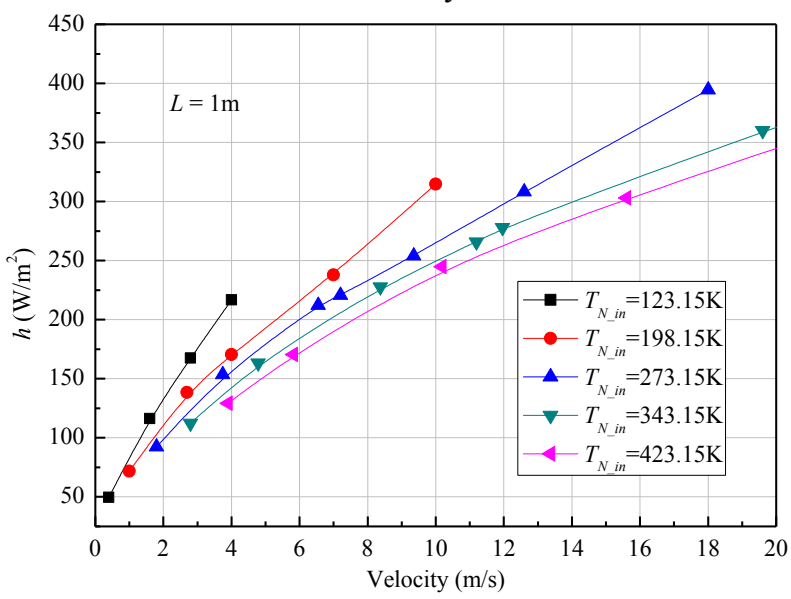

Fig. $6 h$ distribution with varying nitrogen velocity for $L=1 \mathrm{~m}$.

\subsection{Effect of nitrogen temperature}

To get a better understanding of the effects of temperature, the convection heat transfer coefficient at different temperatures for the same flow rate is shown in Fig. 7.

The result shows that the heat transfer coefficient increases with the increasing nitrogen temperature. It is believed that under the same mass flow rate, the number of nitrogen molecules flowing through the shroud surface remains unchanged. With the increase of temperature, the momentum of the nitrogen molecules becomes greater. The more active the molecules are, the more energy exchange between the molecules and the shroud surface happens. In other words, the heat transfer is enhanced as the nitrogen temperature rises for a fixed mass flow rate.

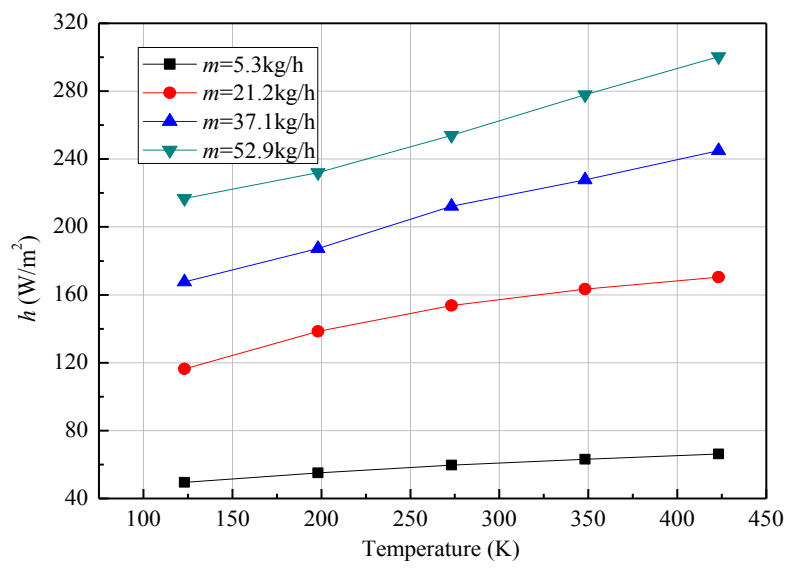

Fig. 7. $h$ distribution with different inlet nitrogen temperature.

\subsection{Dimensionless correlation}

The heat transfer coefficient is influenced by both the inlet velocity and the nitrogen temperature at the same time. Therefore, in order to better understand the relationship between them, the Reynolds number is employed to characterize the flow regime of nitrogen. The calculation formula is as follows.

$$
R e=\frac{\rho u l}{\mu}
$$

where $\rho$ is the nitrogen density and $\mu$ the dynamic viscosity of nitrogen. $l$ is a length scale of the shroud passage, In this paper, the hydraulic diameter of passage cross-section was used as length scale $l$.

Correspondingly, the dimensionless heat transfer characteristics can be represented by Nusselt number.

$$
N u=\frac{h l}{\lambda}
$$

where $\lambda$ is the thermal conductivity of nitrogen. Since $h$ is the average heat transfer coefficient, $N u$ in equation(12) is also an average value for the shroud surface. The curve of $N u$ with $R e$ is shown in Fig. 8.

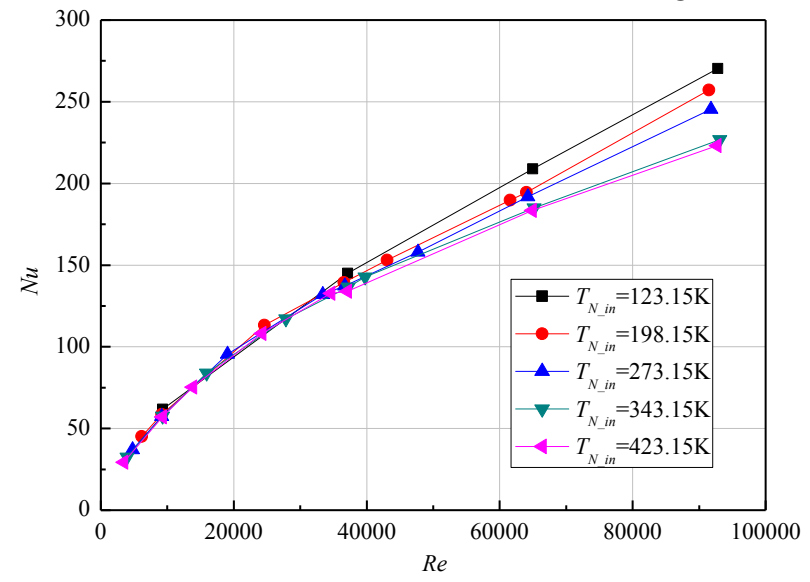

Fig. 8 Average $N u$ with $R e$.

According to the research of Dittus and Boelter [15], the Nusselt number for forced convective heat transfer of fluid can be expressed as a function of Reynolds number and Prandtl number, such as

$$
N u=a e^{b} \operatorname{Pr}^{c}
$$

Based on the numerical results, the correlation equation of the average Nusselt number in terms of Reynolds number and Prandtl number is obtained as follow.

$$
N u=0.23 \operatorname{Re}^{0.63} \operatorname{Pr}^{0.76}
$$

for $3458 \leq R e \leq 93100$ and $123.15 \mathrm{~K} \leq T \leq .423 .15 \mathrm{~K}$.

The calculated results $N u_{C}$ of equation(14) compared with the numerical data $N u_{N}$ are presented in Fig. 9, which shows a very good fitting with the numerical data.

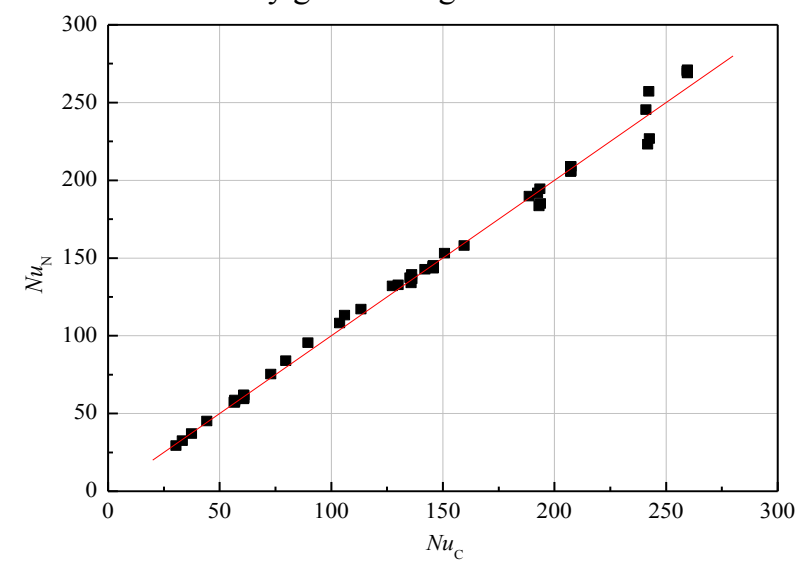

Fig. 9. Comparison between calculated results and numerical data. 


\section{Conclusion}

Extensive numerical study of the heat transfer performance of the GN thermoregulation shroud surface was conducted in this work, where the effects of shroud length, the velocity, the inlet temperature and mass flow rate of nitrogen on average heat transfer coefficient were investigated, and non-dimensional correlation was obtained. The major conclusions of the present study have been summarized as follows:

(1) The length of shroud flow passage has little effect on the heat transfer performance, when the inlet parameters of nitrogen remain unchanged.

(2) The heat transfer coefficient increases with the increase of the velocity under the same inlet temperature. The heat transfer performance is mainly affected by mass flow rate

(3) When the mass flow rate is fixed, the inlet temperature increases heat transfer coefficient as a result of molecular movement.

(4) Based on the numerical data, correlation equation of the average $N u$ was acquired and validated, which is applicable to the $R e$ range from 3458 to 93100 and GN temperature from $123.15 \mathrm{~K}$ to $423.15 \mathrm{~K}$.

Further verification is planned to obtain experimental data and to compare with the present correlation.

\section{References}

1. R. N. Sorge. Space power facility - capabilities for space environmental testing within a single facility. NASA/TM - 2013-217816.

2. J. L. Homan. Creating the deep space environment for testing the James Webb Space Telescope at NASA Johnson Space Center's Chamber A. NASA JSC-CN-28890.

3. D. S. Adams. Mars exploration rover airbag landing loads testing and analysis. 45th AIAA structures, structural dynamics and materials conference. AIAA 2004-1795.

4. G. M. Hill, R. K. Evans. Advanced distributed measurements and data processing at the vibroacoustic test facility, GRC Space Power Facility,
Sandusky, Ohio- an architecture and an example. 25th Aerospace testing conference, (2009).

5. G. Andrina, L Cane, B. Panella. Solar simulation with infrared techniques applicability to Mercury/Bepicolombo probe system thermal test. Proceedings of 4th international symposium on environmental testing for space program, Noordwijk Netherlands, 327-332, (2001).

6. S. G. Price, P. J. Argles. M. Manns. et al. Infra-red thermal balance testing of the Envisat payload module. Proceedings of 4 th international symposium on environmental testiong for space program, Noordwijk Netherlands, 333-340, (2001).

7. A. A. Edwards. Refurbishment of a 39 foot thermal vacuum chamber. NASA-95N14085: 271-297.

8. P. Govindan, M. Satyanarayana, K Deviprasad. et al Design and performance of $0.6 \mathrm{~m}$ thermal vacuum chamber. Proceedings of the 18 th international cryogenic engineering conference, 679-682, (2000).

9. R. N. Watson. A new thermal vacuum facility at Martin Marietta Waterton Plant. 17th Space simulation conference, 32-37, (1992).

10. L. Zhang, M. Liu, Z. Wang, C. He. A review of technologies of temperature-adjustable heat $\operatorname{sink}[\mathrm{J}]$. Spacecraft environment engineering. 29 (2): 179184, (2012).

11. R. N. Watson, G. F. Proulx. A new thermal vacuum facility for Hughes space and communications at EI Swgundo California. 20th Space simulation conference the changing testing paradigm, 113-130, (1998).

12. J. W. Fairbanks, M. B. Eck. A programmable dynamic thermal vacuum system for solar array component testing. NASA-TM-X-66945: 669-682.

13. J. O. Hinze. Turbulence. (New York: McGraw - Hill Publishing Co., 1975).

14. B. E. Launder, D. B. Spalding. Lectures in Mathematical Models of turbulence. (London: Academic Press,1972).

15. F. W. Dittus, L. M. K. Boelter. Heat transfer in automobile radiators of the tubular type. International communications in heat and mass transfer. 12: 3-22, (1985). 